\title{
Control-released basic fibroblast growth factor-loaded poly-lactic-co-glycolic acid microspheres promote sciatic nerve regeneration in rats
}

\author{
HAI-BO SI ${ }^{1,2^{*}}$, YI ZENG $^{1 *}$, YAN-RONG LU ${ }^{2}$, JING-QIU CHENG $^{2}$ and BIN SHEN ${ }^{1}$ \\ ${ }^{1}$ Department of Orthopaedics; ${ }^{2}$ Key Laboratory of Transplant Engineering and Immunology, \\ Regenerative Medicine Research Centre, West China Hospital, Sichuan University, Chengdu, Sichuan 610041, P.R. China
}

Received April 6, 2016; Accepted October 4, 2016

DOI: $10.3892 /$ etm.2016.4013

\begin{abstract}
Although peripheral nerve injury may result in a loss of function in innervated areas, the most effective method for nerve regeneration remains to be determined. The aim of the present study was to investigate the effect of control-released basic fibroblast growth factor (bFGF)-loaded poly-lactic-co-glycolic acid (PLGA) microspheres on sciatic nerve regeneration following injury in rats. bFGF-PLGA microspheres were prepared and their characteristics were evaluated. The sciatic nerve was segmentally resected to create a $10 \mathrm{~mm}$ defect in 36 Sprague Dawley (SD) rats and, following the anastomosis of the nerve ends with a silicone tube, bFGF-PLGA microspheres, free bFGF or PBS were injected into the tube $(\mathrm{n}=12$ in each group). The outcome of nerve regeneration was evaluated using the sciatic function index (SFI), electrophysiological test and histological staining at 6 weeks and 12 weeks post-surgery. The bFGF-PLGA microspheres were successfully synthesized with an encapsulation efficiency of $66.43 \%$. The recovery of SFI and electrophysiological values were significantly greater $(\mathrm{P}<0.05)$, and morphological and histological observations were significantly greater $(\mathrm{P}<0.05)$ in bFGF-PLGA microspheres and bFGF groups compared with those in the PBS group, and the quickest recovery
\end{abstract}

Correspondence to: Professor Jing-Qiu Cheng, Key Laboratory of Transplant Engineering and Immunology, Regenerative Medicine Research Centre, West China Hospital, Sichuan University, 1 Keyuan 4th Road, Chengdu, Sichuan 610041, P.R. China E-mail: jqcheng@scu.edu.cn

Professor Bin Shen, Department of Orthopaedics, West China Hospital, Sichuan University, 37 Guoxue Road, Chengdu, Sichuan 610041, P.R. China

E-mail: shenbin_1971@163.com

*Contributed equally

Key words: basic fibroblast growth factor, control-released, microspheres, nerve regeneration, sciatic nerve was observed in the bFGF-PLGA microspheres group. In conclusion, the bFGF-PLGA microspheres may promote nerve regeneration and functional recovery in the sciatic nerve, and may have potential therapeutic applications in peripheral nerve regeneration.

\section{Introduction}

Peripheral nerve injury is typically caused by accidents, contusion, compression, tumour resection or congenital deformities and may result in total or partial loss of motor, sensory and autonomic functions $(1,2)$. Peripheral nerve injury occurs in $2.8 \%$ of trauma patients (3) and various methods have previously been used to repair this in a clinical setting (4-6). However, the most effective approach for the regeneration of nerve defects remains to be elucidated.

It has been demonstrated that basic fibroblast growth factor (bFGF) is able to promote neurite extension and stimulate Schwann cell proliferation in vivo, and serves an important role in promoting nerve regeneration (7-9). However, the level of endogenous bFGF, which is produced by macrophages, Schwann cells and sensory neurons (2), is typically insufficient for nerve regeneration, and direct delivery of exogenous bFGF is insufficient due to the short half-life and rapid deactivation of bFGF in the body fluids $(10,11)$. It was hypothesised that constructing a controlled-release system may prolong the action time and improve the bioavailability of exogenous bFGF. Due to biological properties such as biocompatibility, bioabsorbency and biodegradability, poly-lactic-co-glycolic acid (PLGA) has been extensively studied for drug delivery, and is employed in several parenteral microspheres in the market (12-16). Therefore, PLGA microspheres may have therapeutic applications in the delivery of a controlled-release matrix of bFGF. In the present study, bFGF-loaded PLGA (bFGF-PLGA) microspheres were developed to bridge a $10 \mathrm{~mm}$ gap in the sciatic nerve, and its effect on sciatic nerve regeneration was evaluated.

\section{Materials and methods}

Preparation of bFGF-PLGA microspheres. PLGA microspheres incorporating bFGF were prepared according to 
previous studies by the present authors $(17,18)$. Briefly, PLGA (molecular weight: $12 \mathrm{kDa}$; 1:1; Chengdu Institute of Organic Chemistry, Chinese Academy of Sciences, Chengdu, China) and freeze-dried bFGF powder (Essex Bio-Technology Co., Ltd., Hong Kong, China) were dissolved in dichloromethane solution, and the mixture was homogenized by sonication into an emulsion, into which $6 \%$ polyvinyl alcohol (PVA) solution was mixed. The mixture was added into PVA solution again and magnetically stirred at room temperature until the dichloromethane was completely volatilized to obtain the bFGF-PLGA microspheres. Finally, $1 \%$ lactose was added as a frame material into the colloid solution of bFGF-PLGA microspheres, which were then freeze-dried.

Characterization of bFGF-PLGA microspheres. The morphology of the bFGF-PLGA microspheres was observed by transmission electron microscopy (H-7500; JEOL, Ltd., Tokyo, Japan). Particle size and distribution were measured using a laser particle size analyzer (Mastersizer 2000; Malvern Instruments, Ltd., Malvern, UK). The drug loading and encapsulation efficiency of bFGF were determined by ELISA, and standard curve method, as described in previous studies by the present authors $(17,18)$. The release ability of the microspheres in vitro was also evaluated. Briefly, $1.5 \mu \mathrm{g}$ bFGF-PLGA microspheres were dissolved in $2 \mathrm{ml}$ PBS at $37^{\circ} \mathrm{C}$ under continuous agitation. At 1-14 days, the solution was centrifuged at $1,500 \mathrm{x} \mathrm{g}$ for $10 \mathrm{~min}$ and the supernatant was collected and analyzed for free bFGF concentration. The dried residues of samples collected at different time points were redissolved in $2 \mathrm{ml}$ PBS for further analysis, and the release curve of bFGF in vitro was plotted.

Rat models. Experiments were performed under a protocol reviewed and approved by the Institutional Ethical Committee of West China Hospital, Sichuan University (Chengdu, China). Rats were housed in a specific pathogen-free environment with a controlled temperature $\left(23-26^{\circ} \mathrm{C}\right)$, humidity $(55-70 \%)$ and diurnal conditions of light/dark (12/12 h). Rats were fed a standard commercial diet and given tap water ad libitum. All rats received humane care, and surgical procedures were performed under sterile conditions with general anaesthesia using intraperitoneal injection of 5\% chloral hydrate $(300 \mathrm{mg} / \mathrm{kg}$; West China Hospital, Sichuan University, Chengdu, China).

A total of 36 female Sprague-Dawley rats (age, $12 \pm 2$ weeks; weight, $250 \pm 20 \mathrm{~g}$ ) were purchased from the Animal Centre of West China Medical Centre, Sichuan University and were randomly divided into the following three groups $(n=12$ in each group): Group A, nerve gap bridged by bFGF-PLGA microspheres; group B, nerve gap bridged by bFGF; and group $\mathrm{C}$, nerve gap bridged by PBS. Following the administration of chloral hydrate, the animal was fixed in a prone position, hair in the surgical area was shaved, and the operative area was disinfected with povidone-iodine solution. A posterior and lateral transgluteal incision was made on the right side, and blunt and acute separation was subsequently performed along the attachment cord between the gluteus maximus and the hipbone to expose the sciatic nerve. At a site $\sim 8 \mathrm{~mm}$ from the lower edge of the piriformis, the sciatic nerve was resected to remove a segment of $\sim 6 \mathrm{~mm}$ and allowed to naturally retract, resulting in a $\sim 10 \mathrm{~mm}$ length of nerve defect (Fig. 1A). Subsequently, a $15 \mathrm{~mm}$ silicone tube was sutured to the proximal and distal ends of the resected sciatic nerve using 8-0 monofilament nylon (Fig. 1B). Following confirmation of the successful completion of anastomosis, $100 \mu \mathrm{l}$ bFGF-PLGA microspheres $(20 \mu \mathrm{g}), 100 \mu \mathrm{l}$ bFGF $(20 \mu \mathrm{g})$ or $100 \mu \mathrm{l}$ PBS was injected into the silicone tube using a microinjector to bridge the nerve gap. Muscle layers and skin were subsequently sutured with 4-0 sutures, and rats were routinely housed following surgery.

Evaluation of sciatic nerve function. The sciatic function index (SFI) was evaluated as described by Bain et al (19). Briefly, the bottom of a wood trough $(100 \times 10 \times 10 \mathrm{~cm})$ with open ends was covered with a clean A4 sheet of white paper and, once the hind limbs were painted with dye, rats were placed in one end of the trough. Rats walked to the other end of the slot and each hind limb left a minimum of five clear footprints. The clear footprints of normal $(\mathrm{N})$ and experimental (E) hind feet were selected and measured for three parameters; print length (PL), toe spread (TS) and intermediary toe spread (IT). The SFI was calculated as follows: SFI=109.5 (ETS-NTS)/NTS-38.3 (EPL-NPL)/NPL+13.3 (EIT-NIT)/NIT-8.8. SFI=0 was considered normal, whereas SFI=100 was considered to indicate full damage.

Neural electrophysiological detection. At six weeks and 12 weeks post-surgery, six rats from each group were anaesthetized as described above. Following re-exposure of the regenerated sciatic nerve, bipolar electrodes were placed mesially at a site $5 \mathrm{~mm}$ proximal to the anastomotic region on the sciatic nerve (the stimulating side) and at a site on the gastrocnemius (the recording side). The nerve conduction velocity (NCV), amplitude of compound muscle action potential (ACMAP) and fractional area (AREA) were recorded with an electromyography system (Keypoint ApS, Frederiksberg, Denmark).

Morphometric and histological analysis. At six weeks and 12 weeks post-surgery, the bridging bodies with $5 \mathrm{~mm}$ of nerve tissues in the proximal and distal ends were harvested and the sciatic nerve tissue of the contralateral site was harvested. The spine was cut to expose the lumbar spinal cord, and the L5 spinal cord and corresponding dorsal root ganglia (DRG) were harvested. These tissues were fixed by neutral paraformaldehyde, dehydrated through grades of ethanol, and embedded in paraffin blocks. The nerve tissues of the mid-bridge body DRG were cut into 5- $\mu \mathrm{m}$ slices, which were subsequently stained with hematoxylin and eosin (H\&E) and observed under a light microscope (AH3; Olympus Corporation, Tokyo, Japan) and $\geq 5$ images of random areas were captured per section. The degree of axon regeneration in each group was evaluated via the mean thickness of myelin sheath, axon diameter, density of myelinated fibre and the survival percentage of DRG neurons, using Mias-2000 software (Sichuan University).

Muscle mass measurement. The weight of the triceps surae (gastrocnemius and soleus) was measured 12 weeks post-surgery. Immediately following sacrifice, the bilateral 

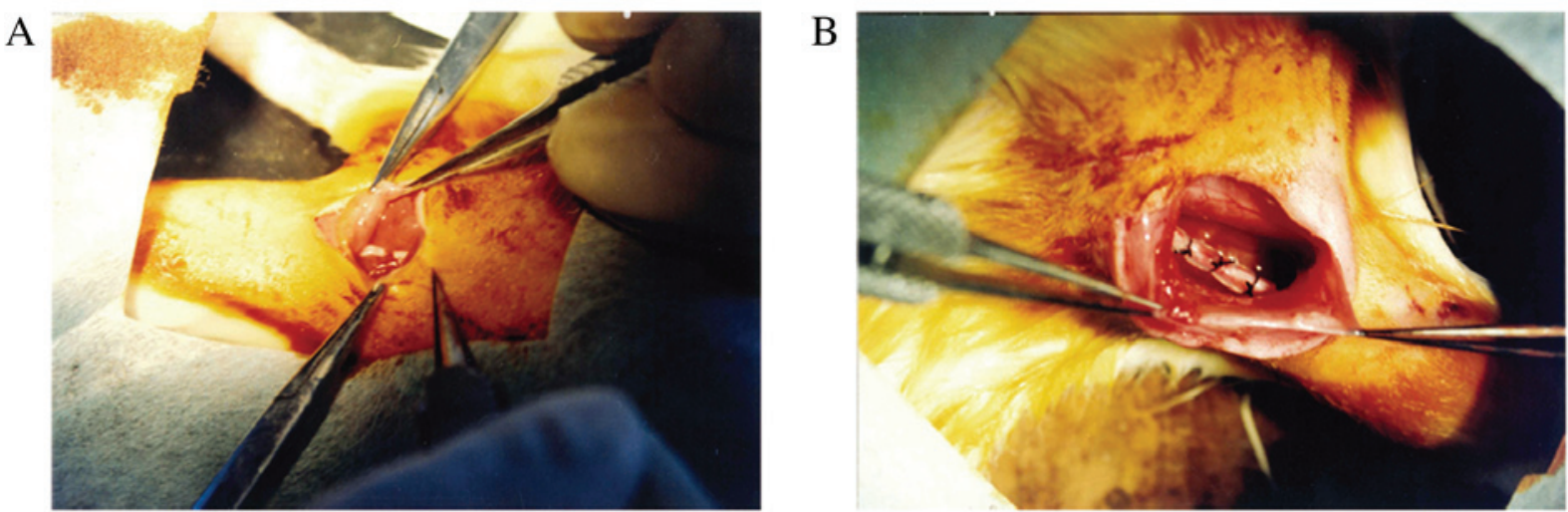

Figure 1. Surgical procedure. (A) At a site $\sim 8 \mathrm{~mm}$ from the lower edge of the piriformis, the sciatic nerve was resected to remove a $\sim 6$ mm segment and allowed to naturally retract, resulting in a $\sim 10 \mathrm{~mm}$ section of nerve defect. (B) A silicone tube 15 mm in length was sutured to the proximal and distal ends of the resected sciatic nerve.

triceps surae were harvested and weighed on an electronic balance (Sartorious AG, Göttingen, Germany) while still wet.

Statistical analysis. All data were expressed as the mean \pm standard error of mean. One-way or two-way analysis of variance was used to compare mean values using the SPSS 22.0 software package (IBM SPSS, Arkmonk, NY, USA). Student-Newman-Kewls post hoc test was used for multiple group comparisons. $\mathrm{P}<0.05$ was considered to indicate a statistically significant difference.

\section{Results}

Characterization of bFGF-PLGA microspheres. Lyophilized powder of the control-released bFGF-PLGA microspheres visibly appeared as uniform, white, loose lumps (Fig. 2A), and as round smooth spheres under a light microscope (Fig. 2B). The mean particle size of bFGF-loaded microspheres was $1.55 \mu \mathrm{m}$, and size variations were distributed normally. The calculated drug-loading rate and encapsulation efficiency of the bFGF-PLGA microspheres were 27.18 and $66.43 \%$ respectively. Within two weeks, the bFGF had been gradually released from the microspheres and the concentration was maintained

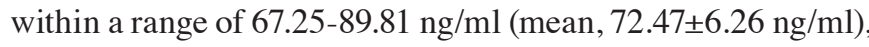
exhibiting a gradual release curve (Fig. 2C), and a release rate of $72.47 \%$ (Fig. 2D).

General conditions of the rat models. Sciatic nerve injury models were successfully established by transection of the sciatic nerve in rats. Prolonged oozing from the wound was observed in two rats, one in group $\mathrm{A}$ and one in group $\mathrm{C}$, however both had healed by two weeks post-surgery. At three weeks post-surgery, plantar ulcers had presented in almost all the rats to varying extents. All ulcers observed in groups A and B had healed by six weeks post-surgery, whereas five had healed in group C. In group C, 2 ulcers remained unhealed at 12 weeks post-surgery, indicating a slower process of reinnervation.

At six weeks and 12 weeks following surgery, no serious adhesions of the regenerated nerve to the surrounding tissue were observed, and there were also no signs of inflammation, granuloma or pseudo neuroma (Fig. 3A). Furthermore, sili- cone tubes were fixed well and there was a thin capsule around the tube (Fig. 3B). At six weeks following surgery, the gap at the injury site was bridged continuously in groups A and B, whereas the proximal and distal ends of the resected sciatic nerve remained separated in three rats in group C. At 12 weeks following surgery, the gaps in all groups were bridged continuously (Fig. 3C).

Functional outcome of the sciatic nerve. The sciatic nerve was damaged completely in each group following the bridging surgery, the majority of SFI values were nearly -100 (group A, -88 to -99 ; group $\mathrm{B},-89$ to -98 ; and group $\mathrm{C},-87$ to -98$)$, and no significant differences were demonstrated among the three groups $(\mathrm{P}>0.05)$. At six weeks post-surgery, varying degrees of neural recovery were observed in each group; compared with group C, the recovery of SFI in groups A and B was significantly quicker $(\mathrm{P}<0.05)$. At 12 weeks following surgery, the increase of SFI in each group continued, with the quickest recovery observed in group A (Fig. 4A).

Results of neural electrophysiological tests. Rats in groups A and B exhibited varying levels of electrophysiological recovery at week six, however there was no marked recovery observed in group C. At 12 weeks post-surgery, rats in all groups showed varying levels of recovery. The NCV in groups A $(34.76 \pm 4.46 \mathrm{~m} / \mathrm{sec})$ and B $(27.12 \pm 3.89 \mathrm{~m} / \mathrm{sec})$ showed significant restoration as compared with group $\mathrm{C}$ $(22.41 \pm 2.24 \mathrm{~m} / \mathrm{sec})(\mathrm{P}<0.05)$. Furthermore, there was a significant difference observed between groups $\mathrm{A}$ and $\mathrm{B}(\mathrm{P}<0.05$; Fig. 4B). ACMAP and AREA results were similar to those of NCV (Fig. 4B).

Morphological and histological results of regenerated nerves. At six weeks post-surgery, H\&E staining indicated that regenerative nerve fibres were growing towards the central area of the silicone tube (Fig. 5A) and the proximal end had merged with the distal end in some samples, particularly in group A (Fig. 5B). At 12 weeks post-surgery, regenerative nerve fibres in group A were markedly the most dense and well arrayed, followed by those in group B, whereas group C nerve fibres displayed the lowest degree of axonal regeneration and littered array (Fig. 5C-F). The myelinated fibre of the regenerated 

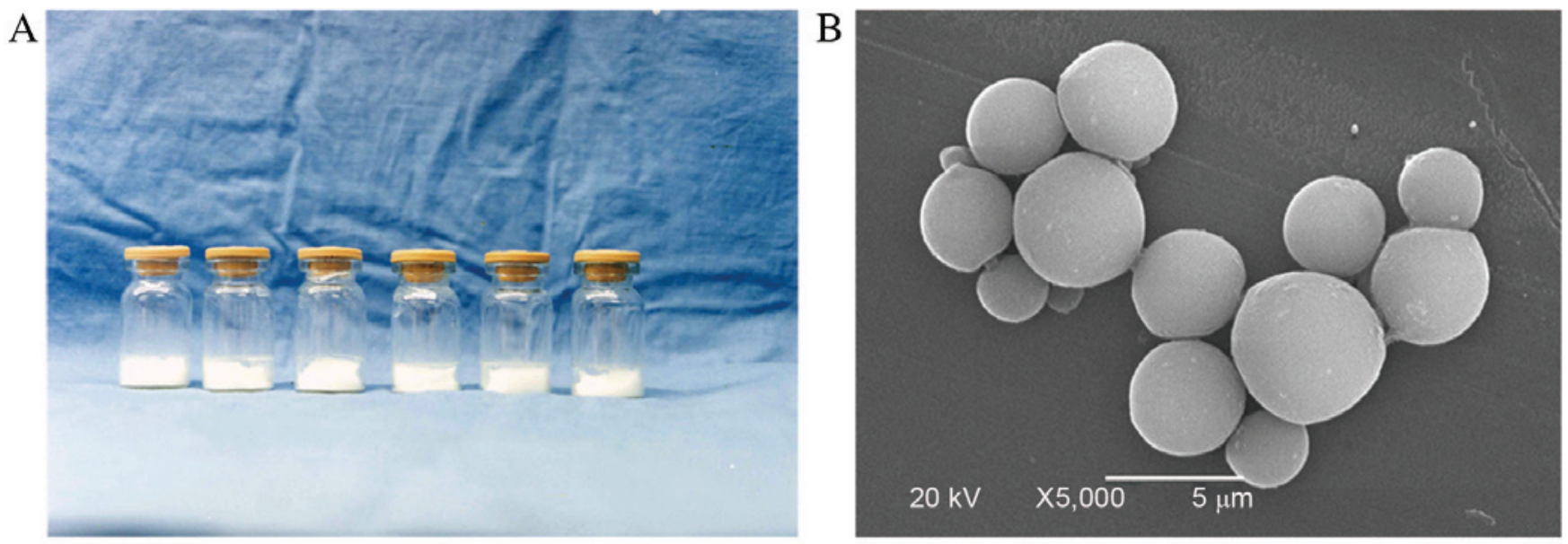

C

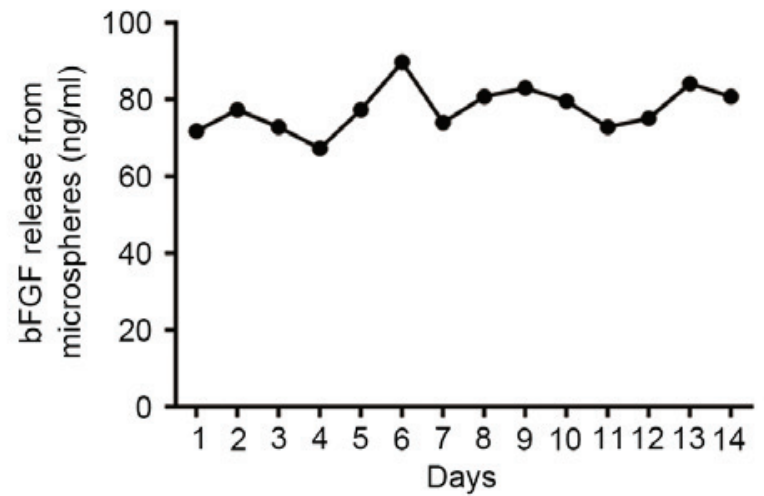

$\mathrm{D}$

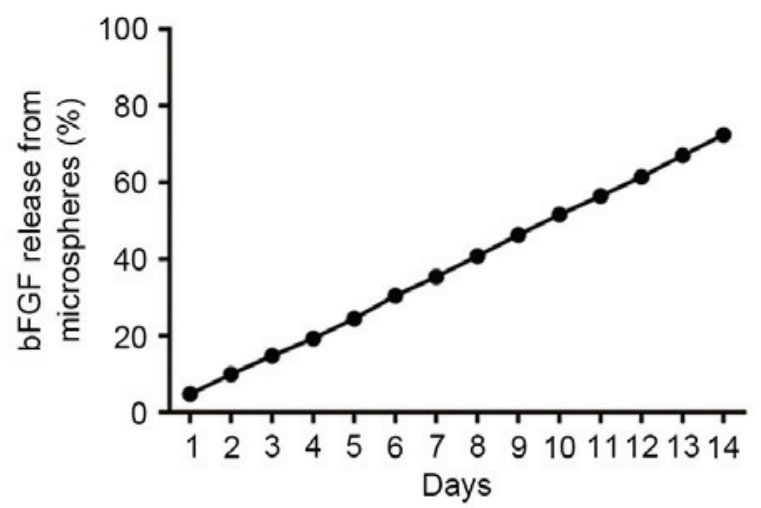

Figure 2. Characteristics of the bFGF-PLGA microspheres. (A) The lyophilized powder of controlled-release bFGF-PLGA microspheres appeared as uniform white loose lumps. (B) The bFGF-PLGA microspheres appeared as round and smooth spheres under transmission electron microscopy (magnification, $\mathrm{x} 5,000$ ). (C) The bFGF was gradually released from the microspheres for 14 days, and the concentration was maintained within a range of 67.25 to $89.81 \mathrm{ng} / \mathrm{ml}$. (D) The slow-release of bFGF-PLGA microspheres displayed a gradual release curve, with a release rate of $72.47 \%$ following 14 days. bFGF, basic fibroblast growth factor; PLGA, poly-lactic-co-glycolic acid.
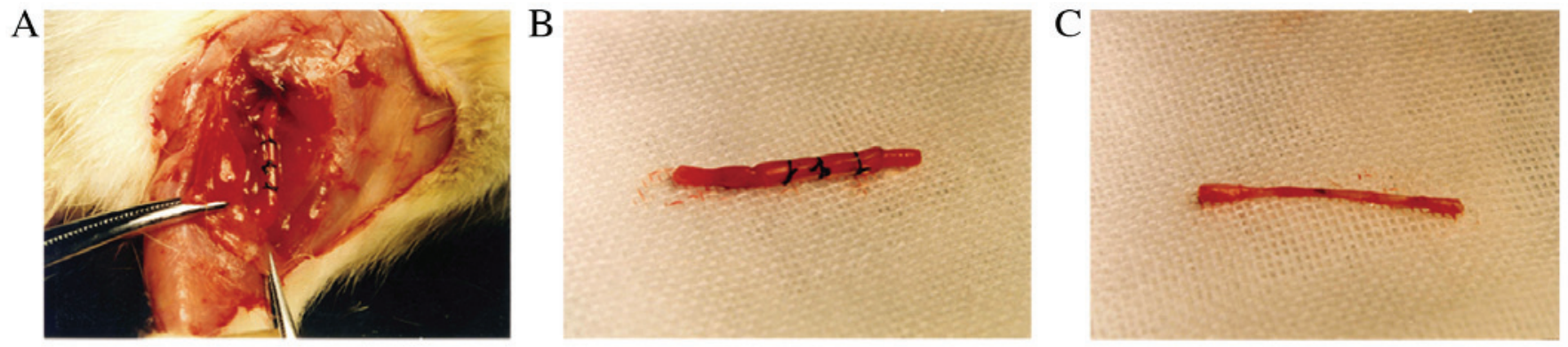

Figure 3. Regeneration of the injured sciatic nerve. (A) No serious adhesion of the regenerated nerve to the surrounding tissue, or signs of inflammation, granuloma or pseudo neuroma, were observed. (B) Silicone tubes were fixed well, and there was a thin capsule around the tube. (C) At 12 weeks post-surgery, gaps in all groups were bridged continuously.

nerve in group A exhibited the thickest myelin sheath, largest axon diameter and highest density (Fig. $5 \mathrm{G}$ and $\mathrm{H}$ ). Neurons in DRG were normal in groups $\mathrm{A}$ and $\mathrm{B}$, although fewer neurons were present in group B (Fig. 6A and B). Fewer neurons were observed in group $\mathrm{C}$ compared with in groups $\mathrm{A}$ or $\mathrm{B}$, and they exhibited an irregular cell shape and cluttered internal structure (Fig. 6C). Furthermore, the highest survival percentage of DRG neurons was observed in group A, followed by groups B and C, respectively, and the differences were statistically significant $(\mathrm{P}<0.05$; Fig. $6 \mathrm{D})$.

Muscle mass measurement. Muscle atrophy may be alleviated upon reinnervation, therefore triceps surae were subjected to muscle mass measurement at week 12. Muscle masses in groups A, B and C were $309.17 \pm 34.39,301.50 \pm 32.39$ and $206.00 \pm 34.72 \mathrm{mg}$, respectively. The muscle mass was 

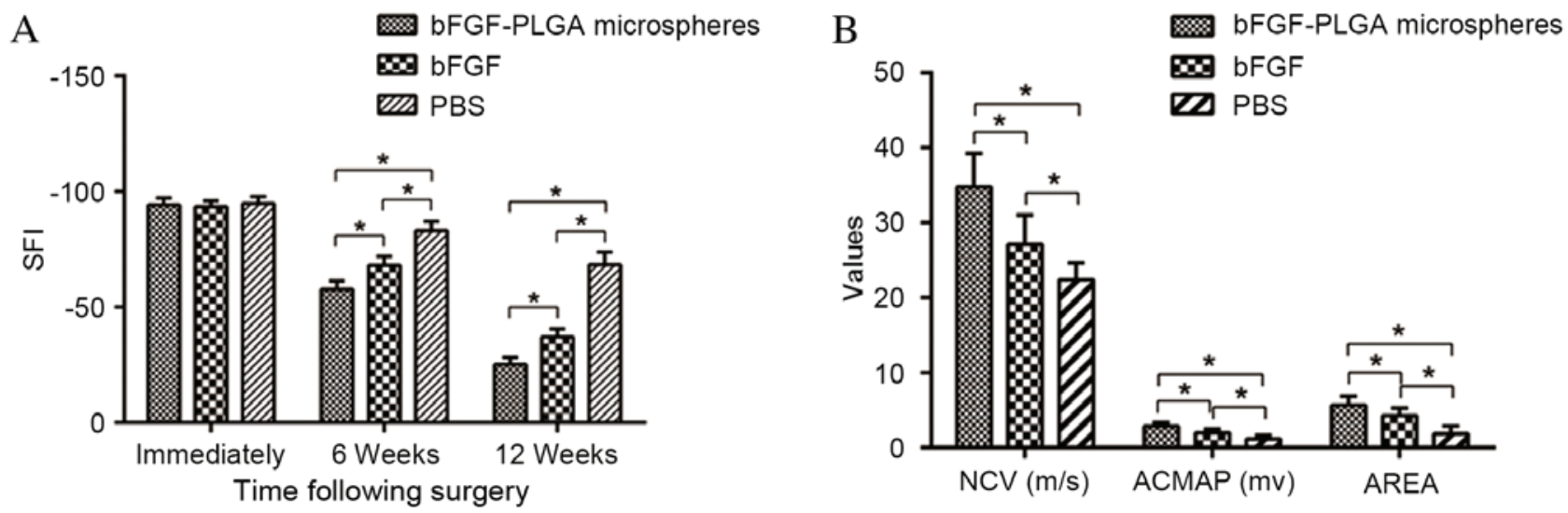

Figure 4. Functional and electrophysiological outcomes of the regenerated nerve fibres. (A) SFI values were nearly all -100 immediately following bridging surgery (group A, -88 to -99 ; group B, -89 to -98 ; and group C, -87 to -98 ), and with no significant differences among the three groups. At 6 and 12 weeks post-surgery, varying degrees of neural recovery were observed in each group, and the recovery of SFI in the bFGF-PLGA microspheres and bFGF groups were quicker when compared with the PBS group. The greatest recovery was observed in the bFGF-PLGA microspheres group. (B) At 12 weeks post-surgery, the NCV, ACMAP and AREA in bFGF-PLGA microspheres and bFGF groups showed significant restoration compared with the PBS group, and there was also a significant difference between the bFGF group and the PBS group "P<0.05. SFI, sciatic function index; bFGF, basic fibroblast growth factor; PLGA, poly-lactic-co-glycolic acid; NCV, nerve conduction velocity; ACMAP, amplitude of compound muscle action potential; AREA, fractional area.
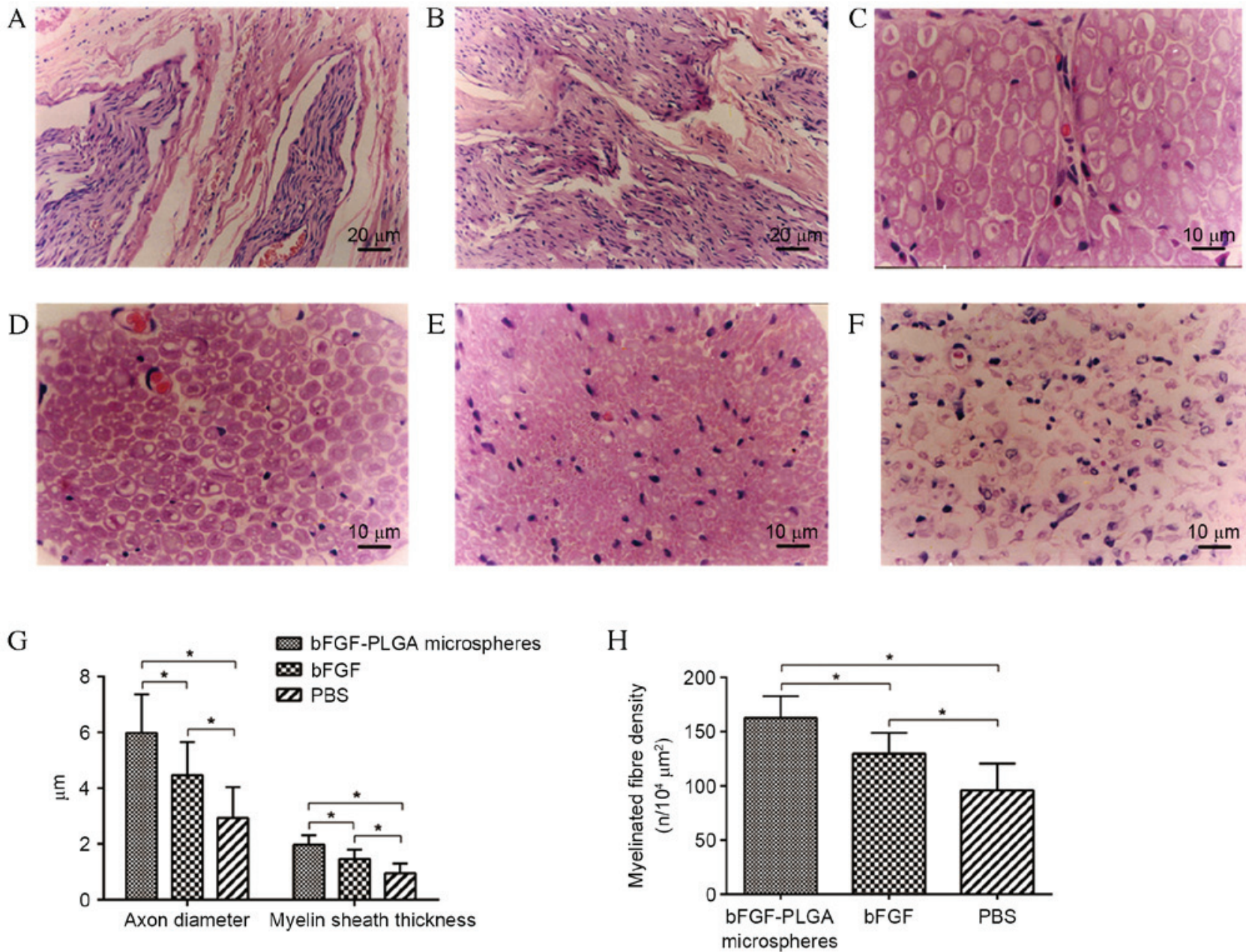

Figure 5. Histological analysis of the regenerative nerve fibres. (A) At six weeks following surgery, regenerative nerve fibres were growing towards the central area of silicone tube. (B) At 6 weeks post-surgery, the proximal nerve had merged with the distal end in some samples, particularly in the bFGF-PLGA microspheres group. (C) Normal sciatic nerve. At 12 weeks post-surgery the regenerative nerve fibres in the (D) bFGF-PLGA microspheres group were markedly the most dense and best arrayed of the groups, followed by the (E) bFGF and (F) PBS groups, which displayed the lowest degree of axonal regeneration and littered array. (G) Comparisons of the thickness of myelin sheath, axon diameter and $(\mathrm{H})$ density of myelinated fibres, indicating that the bFGF-PLGA microspheres group had the thickest myelin sheath, the largest axon diameter and the highest density of myelinated fibres of the regenerated nerve ${ }^{*} \mathrm{P}<0.05$. bFGF, basic fibroblast growth factor; PLGA, poly-lactic-co-glycolic acid. 

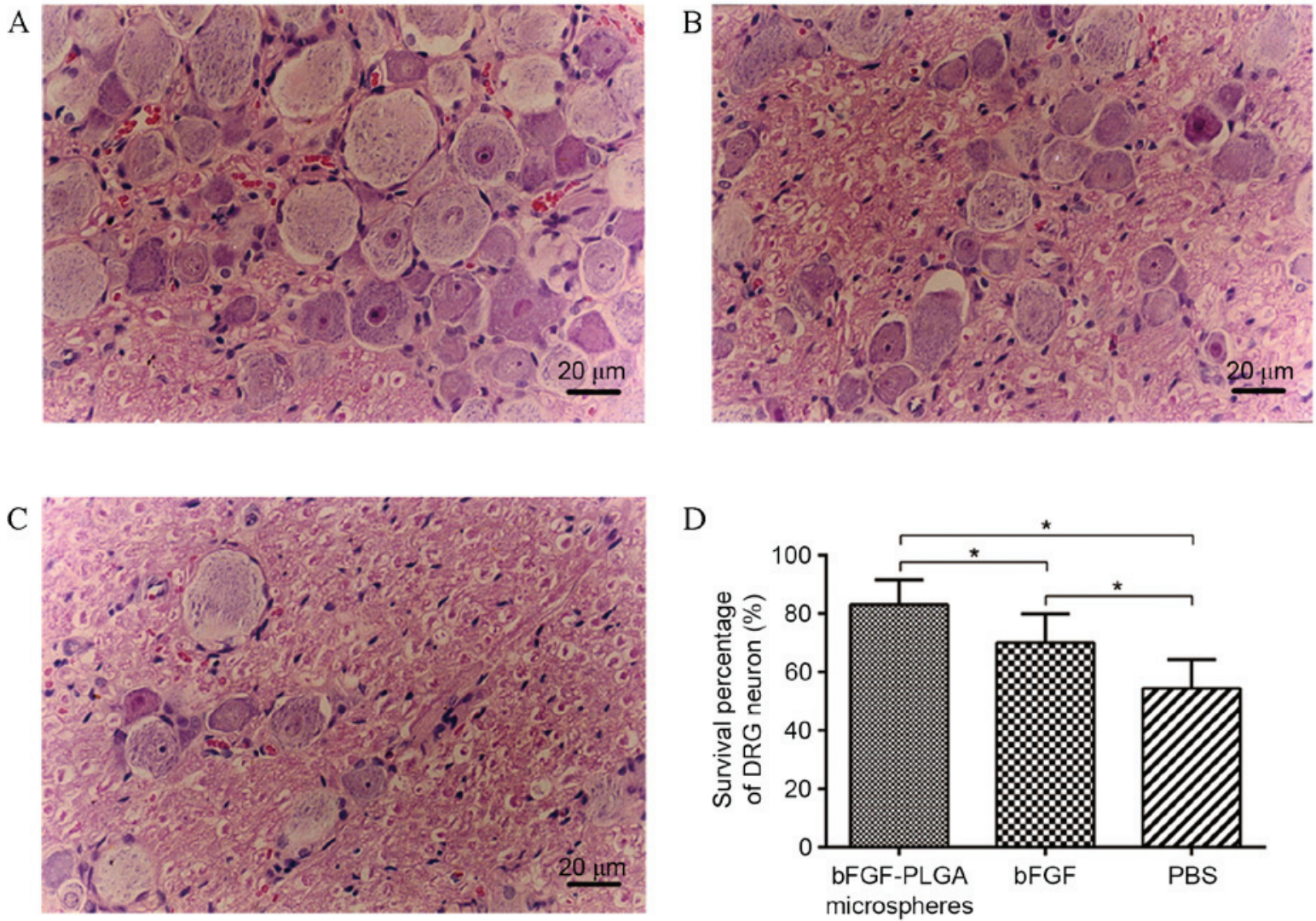

Figure 6. Neurons in the DRG. (A) In the bFGF-PLGA microspheres group, neurons in DRG were distributed evenly and were almost normal in shape and number. (B) In the bFGF group, the number of neurons in DRG was fewer than those in the bFGF-PLGA microspheres group, although the shape was almost normal. (C) In the PBS group, neurons in DRG were markedly thinner and more sparsely distributed, with irregular cell shape, smaller nuclei and more cluttered nucleoli. (D). The highest survival percentage of DRG neurons was observed in the bFGF-PLGA microspheres group, followed by the bFGF and PBS groups. "P<0.05. DRG, dorsal root ganglion; bFGF, basic fibroblast growth factor; PLGA, poly-lactic-co-glycolic acid.

significantly higher in groups A and B compared with in group $C(P<0.05)$, however the difference in mass between groups $\mathrm{A}$ and $\mathrm{B}$ was not statistically significant $(\mathrm{P}>0.05)$.

\section{Discussion}

bFGF is known to stimulate fibroblast proliferation and angiogenesis, and enhance nerve regeneration; however, its application does not always result in successful tissue regeneration due to its short half-life of $\sim 1.5 \mathrm{~min}(7,10,11)$. To overcome this problem, a control-released drug delivery system was introduced. PLGA, approved by the US Food and Drug Administration for in vivo use and clinical trials (20), is a lactic acid and glycolic acid polymer with good biocompatibility and biodegradability in vivo. It degrades slowly via hydrolysis in an aqueous environment with end degradation products of $\mathrm{H}_{2} \mathrm{O}$ and $\mathrm{CO}_{2}$, and has been extensively studied for drug delivery (12-15). Using PLGA microspheres to encapsulate a pharmacological agent may protect it from degradation, postpone the release time and prolong the duration of action $(21,22)$. The present authors have previously demonstrated that the drug loading and encapsulation efficiency of bFGF-PLGA microspheres are significantly higher than those of traditional gelatin microspheres, and that, in vitro, bFGF-PLGA microspheres continuously release bFGF with superior bioactivity to that released from bFGF-PLGA microspheres prepared by the same process (23).
In the present study, bFGF-PLGA microspheres ensured a gradual release of bFGF for $>14$ days, with an overall release rate of $72.47 \%$ at the day 14 , providing a controlled local drug release in the early phase of nerve injury. Although free bFGF was also injected to the local area, it was only able to promote nerve regeneration for a limited time due to the short half-life and rapid deactivation, resulting in nerve regeneration between that of the bFGF-PLGA and PBS groups. These results further confirmed that a local controlled-release of bFGF may provide a conducive microenvironment to allow regenerating axons to cross the defective segment.

Angiogenesis, which occurs during the early stages of traumatic brain injury, stroke and nerve injury, is associated with nerve recovery as sufficient blood supply is a key factor in the creation of an optimal microenvironment for neural regeneration $(24,25)$. Increased angiogenesis during the acute phase following nerve injury is able to stimulate endogenous recovery mechanisms and promote functional recovery (24). Furthermore, vascular endothelial cells are known to secrete certain soluble factors to stimulate neural regeneration, and axonal growth occurs in close correlation with vessels during tissue remodelling following nerve injury (26-28). In the present study, abundant blood vessels were observed around the regenerative nerve in the bFGF-PLGA microspheres group, as bFGF was gradually released into the nerve gap following degradation of the PLGA microspheres. The result of this would be 
persistent stimulation of proliferation of vascular endothelial cells, and further promotion of neural regeneration $(29,30)$.

Guiding of regenerated axons to grow longitudinally through the lesion area is necessary for repairing nerve injury, as linear growth of axons should maintain the native organization and potentially increase the probability of improving functional recovery of the injured nerve (25). Ma et al (27) recently confirmed that scaffolds with longitudinal channels are able to stimulate and guide linear axonal growth following peripheral nerve injury in rats. Various materials have been synthesized with tubular structures or guidance channels to adapt to the anatomy of the peripheral nerve, such as PLGA scaffold (31,32), agarose scaffold (33), collagen scaffold (2) and hyaluronic acid hydrogel scaffold (25). However, this tubular arrangement may be irregular and difficult to reproduce. The use of a silicone tube to bridge the gap in the rat sciatic nerve in the present study is a simple and highly reproducible solution, as described previously (34). Notably, the silicone tube seems to exhibit an affinity for the guidance of axonal growth through the channel, as has been observed visibly and via histological analysis, particularly in the bFGF-PLGA microspheres group.

In conclusion, the bFGF-PLGA microspheres employed in the present study were able to gradually release bFGF in the early phase of sciatic nerve regeneration in rats, which leads to accelerated nerve regeneration and functional recovery. The use of bFGF-PLGA microspheres may be a promising and effective approach for peripheral nerve regeneration.

\section{Acknowledgements}

The authors wish to thank Professor Zong-ke Zhou and Professor Fu-xing Pei at West China Hospital, Sichuan University, who read the manuscript and helped to improve it.

\section{References}

1. Kline DG, Kim D, Midha R, Harsh C and Tiel R: Management and results of sciatic nerve injuries: A 24-year experience. J Neurosurg 89: 13-23, 1998.

2. Ma F, Xiao Z, Chen B, Hou X, Dai J and Xu R: Linear ordered collagen scaffolds loaded with collagen-binding basic fibroblast growth factor facilitate recovery of sciatic nerve injury in rats. Tissue Eng Part A 20: 1253-1262, 2014.

3. Noble J, Munro CA, Prasad VS and Midha R: Analysis of upper and lower extremity peripheral nerve injuries in a population of patients with multiple injuries. J Trauma 45: 116-122, 1998.

4. Chen B, Niu SP, Wang ZY, Wang ZW, Deng JX, Zhang PX, Yin XF, Han N, Kou YH and Jiang BG: Local administration of icariin contributes to peripheral nerve regeneration and functional recovery. Neural Regen Res 10: 84-89, 2015.

5. Ding T, Zhu C, Yin JB, Zhang T, Lu YC, Ren J and Li YQ: Slow-releasing rapamycin-coated bionic peripheral nerve scaffold promotes the regeneration of rat sciatic nerve after injury. Life Sci 122: 92-99, 2015.

6. Ikeguchi R, Kakinoki R, Matsumoto T, Yamakawa T, Nakayama K, Morimoto Y, Tsuji H, Ishikawa J and Nakamura T: Basic fibroblast growth factor promotes nerve regeneration in a C-ion-implanted silicon chamber. Brain Res 1090: 51-57, 2006.

7. Ikeda M, Uemura T, Takamatsu K, Okada M, Kazuki K, Tabata Y, Ikada Y and Nakamura H: Acceleration of peripheral nerve regeneration using nerve conduits in combination with induced pluripotent stem cell technology and a basic fibroblast growth factor drug delivery system. J Biomed Mater Res A 102: 1370-1378, 2014

8. Matsumine H, Sasaki R, Tabata Y, Matsui M, Yamato M, Okano T and Sakurai H: Facial nerve regeneration using basic fibroblast growth factor-impregnated gelatin microspheres in a rat model. J Tissue Eng Regen Med 10: E559-E567, 2014.
9. Fujimoto E, Mizoguchi A, Hanada K, Yajima M and Ide C: Basic fibroblast growth factor promotes extension of regenerating axons of peripheral nerve. In vivo experiments using a Schwann cell basal lamina tube model. J Neurocytol 26: 511-528, 1997.

10. Chen B, He J, Yang H, Zhang Q, Zhang L, Zhang X, Xie E, Liu C, Zhang R, Wang Y, et al: Repair of spinal cord injury by implantation of bFGF-incorporated HEMA-MOETACL hydrogel in rats. Sci Rep 5: 9017, 2015.

11. Ohta M, Suzuki Y, Chou H, Ishikawa N, Suzuki S, Tanihara M, Suzuki Y, Mizushima Y, Dezawa $M$ and Ide C: Novel heparin/alginate gel combined with basic fibroblast growth factor promotes nerve regeneration in rat sciatic nerve. J Biomed Mater Res A 71: 661-668, 2004.

12. Makadia HK and Siegel SJ: Poly Lactic-co-Glycolic Acid (PLGA) as Biodegradable Controlled Drug Delivery Carrier. Polymers (Basel) 3: 1377-1397, 2011.

13. Danhier F, Ansorena E, Silva JM, Coco R, Le Breton A and Préat V: PLGA-based nanoparticles: An overview of biomedical applications. J Control Release 161: 505-522, 2012.

14. Gu B and Burgess DJ: Prediction of dexamethasone release from PLGA microspheres prepared with polymer blends using a design of experiment approach. Int J Pharm 495: 393-403, 2015.

15. Xie X, Lin W, Xing C, Yang Y, Chi Q, Zhang H, Li Y, Li Z, Yang $\mathrm{Y}$, Yang $\mathrm{Z}$ and $\mathrm{Li} \mathrm{M}$ : In vitro and in vivo evaluations of PLGA microspheres containing nalmefene. PLoS One 10: $\mathrm{e} 0125953,2015$.

16. Guo J, Wu SH, Ren WG, Wang XL and Yang AQ: Anticancer activity of bicalutamide-loaded PLGA nanoparticles in prostate cancers. Exp Ther Med 10: 2305-2310, 2015.

17. Shen B, Duan H, Chen J, Yang J and Pei F: Preparation of controlled release microsphere incorporating bFGF and its effect on Schwann cells. Sheng Wu Yi Xue Gong Cheng Xue Za Zhi 22: 719-724, 2005 (In Chinese)

18. Shen B, Pei FX, Duan H, Chen J and Mu JX: Preparation and in vitro activity of controlled release microspheres incorporating bFGF. Chin J Traumatol 11: 22-27, 2008.

19. Bain JR, Mackinnon SE and Hunter DA: Functional evaluation of complete sciatic, peroneal, and posterior tibial nerve lesions in the rat. Plast Reconstr Surg 83: 129-138, 1989.

20. Choi YH, Heo SC, Kwon YW, Kim HD, Kim SH, Jang IH, Kim JH and Hwang NS: Injectable PLGA microspheres encapsulating WKYMVM peptide for neovascularization. Acta Biomater 25: 76-85, 2015.

21. Huang W, Shi X, Ren L, Du C and Wang Y: PHBV microspheres-PLGA matrix composite scaffold for bone tissue engineering. Biomaterials 31: 4278-4285, 2010.

22. Choi YS, Park SN and Suh H: Adipose tissue engineering using mesenchymal stem cells attached to injectable PLGA spheres. Biomaterials 26: 5855-5863, 2005.

23. Shen B, Pei FX, Chen J and Duan H: Effect of controlled release microspheres incorporating bFGF on Schwann cells. Sichuan Da Xue Xue Bao Yi Xue Ban 36: 873-876, 2005 (In Chinese).

24. Sekiguchi H, Ii M, Jujo K, Thorne T, Ito A, Klyachko E, Hamada H, Kessler JA, Tabata Y, Kawana M, et al: Estradiol promotes neural stem cell differentiation into endothelial lineage and angiogenesis in injured peripheral nerve. Angiogenesis 16: 45-58, 2013.

25. Wen Y, Yu S, Wu Y, Ju R, Wang H, Liu Y, Wang Y and Xu Q: Spinal cord injury repair by implantation of structured hyaluronic acid scaffold with PLGA microspheres in the rat. Cell Tissue Res 364: 17-28, 2016.

26. Shen Q, Goderie SK, Jin L, Karanth N, Sun Y, Abramova N, Vincent P, Pumiglia K and Temple S: Endothelial cells stimulate self-renewal and expand neurogenesis of neural stem cells. Science 304: 1338-1340, 2004.

27. Ma F, Xiao Z, Meng D, Hou X, Zhu J, Dai J and Xu R: Use of natural neural scaffolds consisting of engineered vascular endothelial growth factor immobilized on ordered collagen fibers filled in a collagen tube for peripheral nerve regeneration in rats. Int J Mol Sci 15: 18593-18609, 2014.

28. Moimas S, Novati F, Ronchi G, Zacchigna S, Fregnan F, Zentilin L, Papa G, Giacca M, Geuna S, Perroteau I, et al: Effect of vascular endothelial growth factor gene therapy on post-traumatic peripheral nerve regeneration and denervation-related muscle atrophy. Gene Ther 20: 1014-1021, 2013.

29. Tanihara M, Suzuki Y, Yamamoto E, Noguchi A and Mizushima Y: Sustained release of basic fibroblast growth factor and angiogenesis in a novel covalently crosslinked gel of heparin and alginate. J Biomed Mater Res 56: 216-221, 2001. 
30. Kigami R, Sato S, Tsuchiya N, Sato N, Suzuki D, Arai Y, Ito K and Ogiso B: Effect of basic fibroblast growth factor on angiogenesis and bone regeneration in non-critical-size bone defects in rat calvaria. J Oral Sci 56: 17-22, 2014.

31. Du C, Yao C, Li N, Wang S, Feng Y and Yang X: Cell sheet-engineered bones used for the reconstruction of mandibular defects in an animal model. Exp Ther Med 10: 2216-2220, 2015.

32. Gu Y, Zhu W, Hao Y, Lu L, Chen Y and Wang Y: Repair of meniscal defect using an induced myoblast-loaded polyglycolic acid mesh in a canine model. Exp Ther Med 3: 293-298, 2012.
33. Stokols S and Tuszynski MH: Freeze-dried agarose scaffolds with uniaxial channels stimulate and guide linear axonal growth following spinal cord injury. Biomaterials 27: 443-451, 2006.

34. Dahlin LB, Anagnostaki L and Lundborg G: Tissue response to silicone tubes used to repair human median and ulnar nerves. Scand J Plast Reconstr Surg Hand Surg 35: 29-34, 2001. 